\title{
Nature's Swell, But Is It Worth Copying? Steven Vogel
}

The following article is based on the plenary presentation by Steven Vogel (Duke University), delivered at the 2002 Materials Research Society Fall Meeting on December 2 in Boston.

"Biomimetics" has become a buzzword $d u$ jour; perhaps not as buzzy as, say, "bioinformatics," but something to conjure with-in particular, something with which to conjure coinage. It has rapidly acquired a community with no small stake in its promise, so it is worth examining with if not a skeptical then at least a detached eye. How much is reality? How much comes down to mere hype and hope?

Consider the following quotation, by Dorion Sagan in a review of Janine Benyus's book Biomimicry, in the New York Times Book Review (August 31, 1997): "...[Biomimicry] shows us once and for all the utter technological superiority of would-be 'lower' life forms: the underwater superglue made by mussels; a spider's dragline silk that, ounce for ounce, is five times as strong as steel and five times as impact-resistant as bulletproof Kevlar...." That is no straw man, eitherI have a small file of such claims of nature's natural superiority or perfection, dating from Aristotle to the present. ${ }^{1}$

\section{In Defense of the Unnatural}

The engineer or materials scientist can dismiss such anti-technological posturing with neither guilt nor hubris. Evolutionary theory provides no justification for assuming perfection in natural design-we see only a blundering trial-and-error process working against a moving target, both physical and biological. Natural selection suffers from lack of foresight, near impossibility of cross-lineage transfer of innovations, great difficulty making anything but incremental alterations, severe lock-in of established if fundamentally inferior designs, unavoidably multifunctional devices, and limitation to locally available

Material Matters is a forum for expressing personal points of view on issues of interest to the materials community. resources, just to mention a few of the constraints under which it labors.

Nor do these impose merely hypothetical limitations, ones more than offset by the time nature has at its disposal for testing and altering. Consider just a few items that give splendid service in human technology but which never (or hardly ever) appear in the technology of nature: Our wheel-and-axle devices, meaning not just wheeled vehicles, but all our rotating pulleys, gears, cams, and capstans, have no macroscopic equivalent. Every cell has the enzymatic machinery to handle metal atoms, and metal salts harden lots of structures, but no metallic material appears, as far as we know, in any organism. Our long-used trick of weaving threads in some orthogonal array to make, in essence, a one-component composite, has no analogue-nature inevitably glues fibers together or compresses unglued layers. Only we do the fine trick of spinning long threads or ropes from short fibers, capitalizing on the radial compression induced by axial tension in helical windings and on interfiber shear resistance. Only we make lighter-than-air fliersblimps and balloons; "ballooning" in nature amounts to little more than capitalizing on high drag. Only we make thermal engines, which incidentally can achieve much better efficiencies and power-toweight ratios than nature's best-muscle. And so on. ${ }^{1}$

Even the highly touted superiority of spider silk turns out to be if not entirely mythological then at least misleading. Table I puts some numbers on the comparison, ignoring for simplicity the diversity of both steels and silks.

Table I: Typical Mechanical Properties of Three Kinds of Materials. ${ }^{2}$

\begin{tabular}{lccc}
\hline & Kevlar & Steel & Spider Silk \\
Young's modulus (GPa) & 130 & 200 & 10 \\
Tensile strength (GPa) & 3.6 & 2.0 & 2.0 \\
Extensibility & 0.027 & 0.2 & 10 \\
Work of extension $\left(\mathrm{MJ} / \mathrm{m}^{3}\right)$ & 40 & 1.0 & 200 \\
Work of extension $(\mathrm{J} / \mathrm{kg})$ & 30,000 & 150 & 160,000 \\
$\quad$ (same, relative to mass) & & & \\
\hline
\end{tabular}

What we see is not so much superiority as eclecticity. Silk's high work of extension comes mainly from its extreme extensibility rather than from its strength, and with that low stiffness and high extensibility comes the expected high work of extension. But the work of extension cannot be recovered mechanically, so low is silk's resilience-a mere $35 \%$. It is great stuff for a web-a very low-mass, low-visibility catch-net suitable for stopping and holding prey that fly in with lots of momentum, prey that might break fibers were it not for that tarbaby-like extensibility, or be flung out again were it not for that low resilience. It is much like a spring and dashpot in parallel, not something we are used to seeing in a single material.

Nor must one focus on some specific material to debunk the notion of nature's natural preeminence. Figure 1 gives a view of two critical but commonly conflicting material properties-work of fracture (often termed "toughness") and Young's modulus of elasticity (loosely, "stiffness"). Biological materials span a wide range of Young's moduli, from mesoglea (essentially, jellyfish jelly), to such things as coral and tooth enamelabout as wide a range as our technological materials. Biological materials' works of fracture vary relatively less, and-a bit oddly-they appear constrained to gain excellence in one property at the expense of diminished performance in the other. If engineering materials face a similar limitation, they certainly face a much ameliorated version of it, with a wide variety of both metals and nonmetals far above the biological line. Biology bests inanimate nature, but even the materials of ancient technology beat the living world.

Dismissing natural preeminence as technophobic mythology might imply that biomimetics is a fool's errand, an odd manifestation (or capitalization on) a current of cultural pantheism. Paradoxically, perhaps, that dismal corollary proves spurious. It would hold true only if nature's technology were no more than an inferior version of our own, a more constrained manifestation of the single 


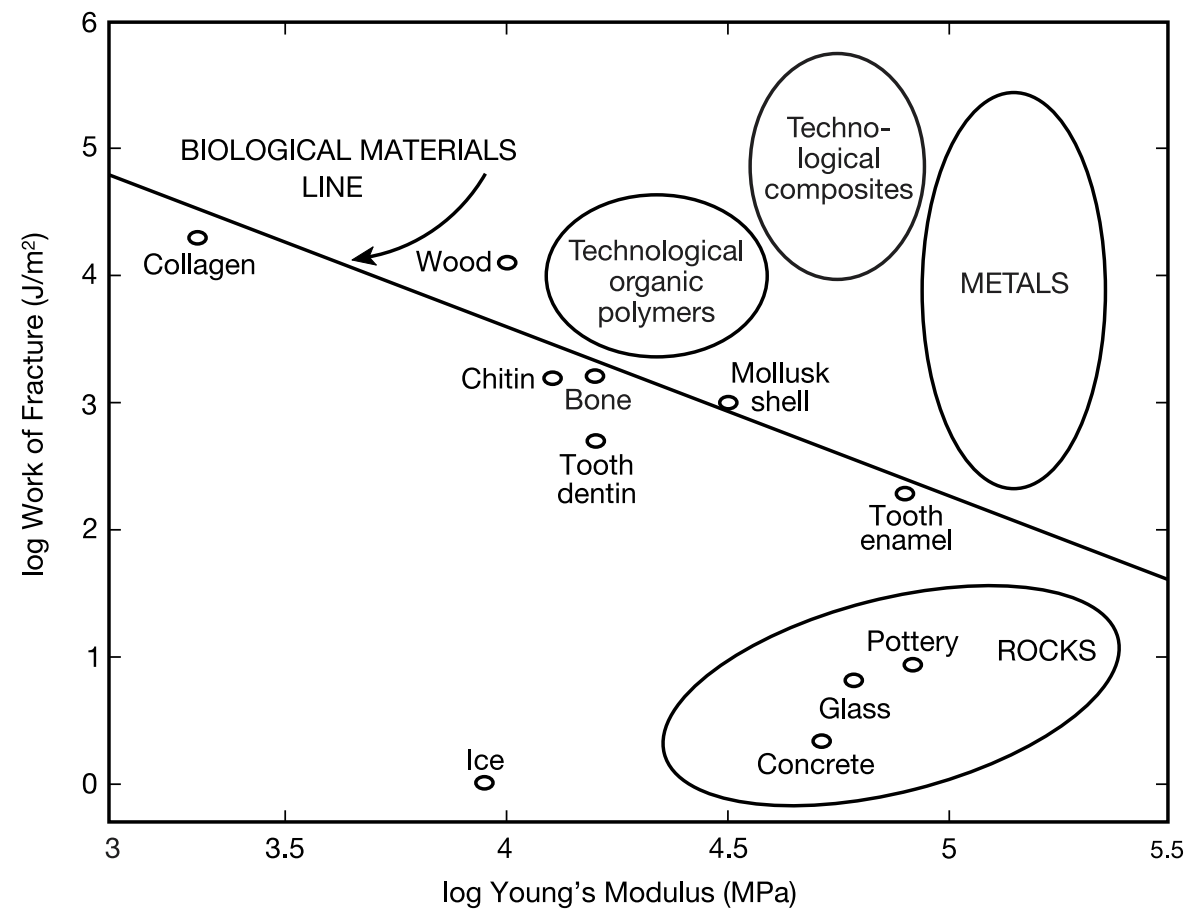

Figure 1. Young's modulus of elasticity versus work of fracture for a wide variety of living, nonliving, and manufactured materials.

technology possible given the constituents and physical conditions of our planet. In fact, nature's technology differs from our own along a host of axes, and in those differences lies the promise of biomimetics. This last assertion might be best supported with examples. We will first consider a few low-stiffness designs and then some anisotropic composites.

\section{Where Strength, Not Stiffness, is the Criterion}

Nature, as James Gordon noted, cares more often about strength than about stiffness. ${ }^{3}$ Tolerance of flexibility should permit the use of less material. One can, though, recognize further implications. In particular, many of nature's designs do not merely tolerate low elastic moduli, but capitalize upon them in diverse ways.

A tall sea anemone, Metridium senile, extracts suspended edibles from water passing through its bushy crown of tentacles (Figure 2). For that crown to do its task, it has to be well above the substratum and normal to whatever happens to be the direction of flow at any particular moment. A universal joint just beneath the crown ensures that orientation, taking advantage of the drag of the crown to power the movement. That joint consists of nothing more than an apical narrowing of the anemone's columnar body, taking advantage of the dependence of flexural stiffness on the second moment of area. In no way does the material of the body, mesoglea, differ at the joint from elsewhere. ${ }^{4}$

Another coelenterate, the marine hydroid Abietenaria, consists of a twodimensional array of tiny polyps on the branches of a stalk-looking, overall, like a fern leaf. Like Metridium, it is a suspension feeder faced with variable flows. The polyps feed most effectively in flows of $2-3 \mathrm{~cm} / \mathrm{s}$; flexibility of the stalk ensures

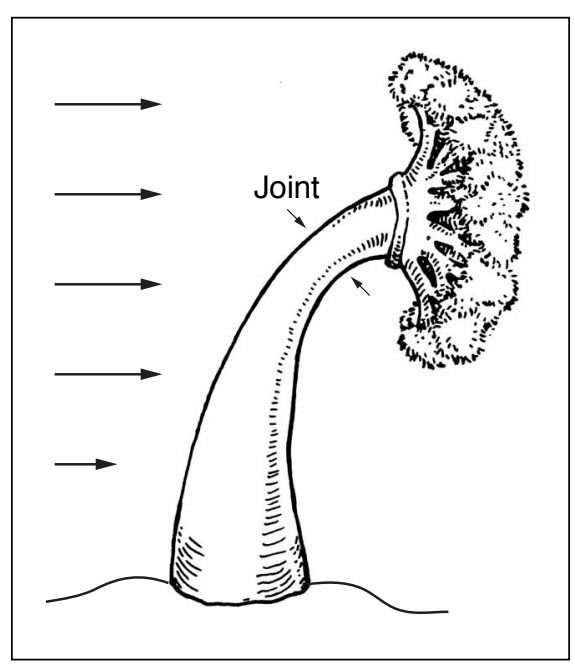

Figure 2. The sea anemone Metridium senile, bent beneath the crown by the current. sufficient flow-dependent bending so that flow passing individual polyps stays in that speed range even as the overall flow reaches a speed an order of magnitude faster. ${ }^{5}$

A tube-making infaunal marine worm, Eupolymnia, sticks a host of long, thin, feeding tentacles out into a passing flow. These tentacles fan out and attach, near their ends, to pebbles and similar fixed elevations above the main substratum (Figure 3 ). The tentacles experience drag from the current, but by stretching in the flow, they gain the advantage of a rope between two supports that sags downward instead of being pulled taut - the greater the sag, the less the stress. But, unlike what our relatively inextensible ropes would do, tentacle sag depends on flow speed. Thus tentacles feel a tensile stress that rises little with increasing flow speed. ${ }^{6}$

A mussel, Mytilus edulis, lives on rocks in very rapid flows. In dense arrays, each individual hangs on with a bunch of fine ropes, the "beard" of byssus threads that we remove in the kitchen. Both rock and mussel shell lack flexibility, so load sharing can be achieved only through tuning of the properties of the threads. These consist of collagen, the material of our tendons, but an especially extensible kind of collagen (which is ordinarily fairly stiff). The portion of each thread farthest from the shell yields just short of the breaking force. That permits threads to reorient themselves closer to the direction of the applied force and to share increasing loads among an increasing number of threads. ${ }^{7}$

The drag of a rigid, unstreamlined body commonly varies with (roughly) the square of speed. A wide variety of sessile organisms that face severe flows manage to do better, indeed even better quite often than does a streamlined body, whose drag increases with speed to a power between 1.5 and 1.8. Values for that exponent run from about 0.5 to 1.5 the first describes a situation in which drag varies with the square root, not the

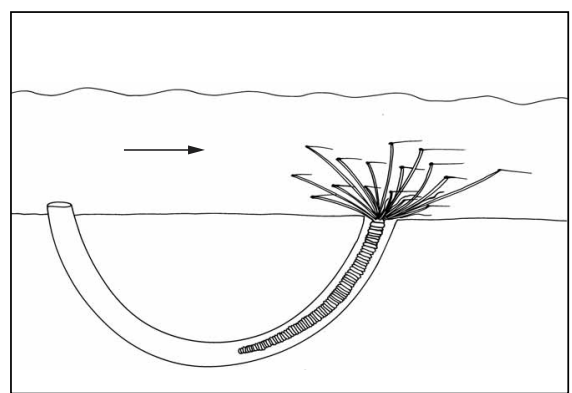

Figure 3. The tubicolous marine worm Eupolymnia, with its tentacles extended across the current. 
square of speed. What these sessile organisms have in common is flexibility. What they do in common can best be described as reconfiguring in flow ("deforming" sounds all too pathological). ${ }^{8}$ A leaf on a tree in a storm (Figure 4 ) can afford some short-term sacrifice of photosynthesis (since sunlight in storms is in short supply anyway), as can an alga on a rock when hit by a wave.

\section{Twisting in the Wind}

Flexibility, as one might guess from the mussel's trick, is no simple, linear variable, and stress-strain curves can take many forms. Sometimes a lot of area underneath gives something desirableas when, in running, we use our tendons for stride-to-stride elastic-energy storage. Sometimes minimal area does better-as in our arterial walls, which have to smooth the fluctuating pressures of the cardiac pump with some aneurysmdefeating increase in stiffness as extension proceeds. ${ }^{1}$

For that matter, a single stress-strain plot will not suffice, even where viscoelasticity plays no role. Flexibility can turn on geometric as well as material variables, as in the anemone's joint rather than the mussel's thread. But even the paired components of flexural stiffness are not enough. Nature appears to manipulate torsional stiffness as well, so we need to look at cross-sectional shape in yet another way, and we have to consider shear modulus. Again, we are accustomed to designing stiff items, providing sufficient torsional stiffness to prevent, say, a driveshaft from twisting too much under load. We commonly use I-beams, structures of low torsional stiffness, but we use them in parallel pairs or groups, so one offsets that disability of another.

Nature makes widespread use of structures of low torsional stiffness, by contrast capitalizing on just that flexibility. A useful variable for assessing biological designs is the ratio of flexural to torsional stiffness-EI/GJ in the usual notation, and "twistiness-to-bendiness" in handy vernacular. For a circular cylinder of metal, the value is about 1.2; for an isovolumetric material, it is 1.5. Deviation from circularity raises the value, with a triangular isovolumetric section giving 2.7. What of the stem (petiole) of a leaf, which has to be flexurally stiff as the blade-supporting cantilever, but torsionally flexible enough to permit multileaf clustering in a storm? Leaf stems run between about 3 and 6 , with sections that deviate only slightly from circularity. ${ }^{9}$

What about the primary (tip) wing feathers of a bird, from which the bird

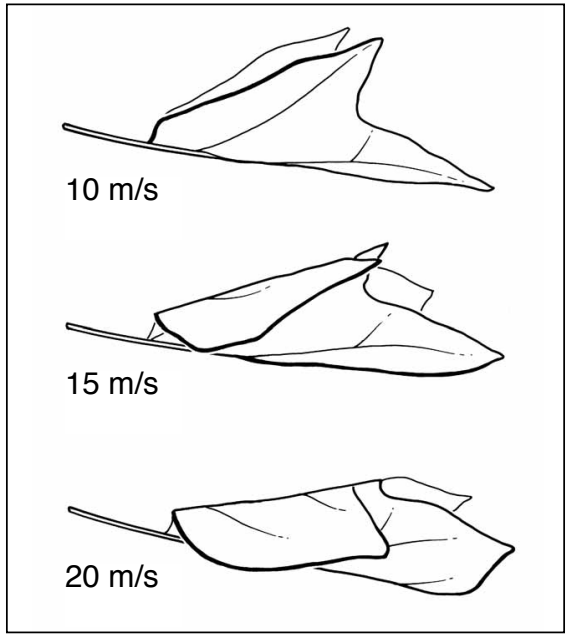

Figure 4. A tulip poplar leaf reconfigures itself in an increasing wind.

hangs in flight but that must twist one way during upstroke, the other way during downstroke? What little data we have suggest values around 5. Tree trunks, which will ordinarily experience twisting as well as bending loads in high winds, have values running between about 6 and 10, despite being almost perfectly round in section. One can rationalize the result in functional terms by noting that twisting might actually reduce flexural loading as part of a large-scale reconfiguration of a storm-challenged tree. Nor are these truly extremes-that, at present, seems to be the long, vertical stalk of a sedge plant, whose head swings downwind in a breeze; a sedge stalk can have a twistiness-to-bendiness ratio of 25 to $50 .{ }^{10}$
Twisting as a means of reducing flexural loading certainly happens in daffodil stems. Flower heads in a wind tunnel experience $30 \%$ less drag when facing away from the wind than into it, and flowers on their stems do, like sedges, swing around to face downwind. Facilitating that torsional motion is an $E I / G J$ of 13 , an order of magnitude above the value for a circular metallic cylinder. Their peculiar flexibility, in concert with the irregularity of groundlevel breezes, forms the biomechanical basis for daffodils' habit of "dancing" in the wind, behavior to which literary allusions abound. ${ }^{11}$

With the exception of the tree trunks, all these twisty structures-feathers, stems, petioles, stalks-have noncircular cross sections, as shown in Figure 5. But in all of these cases, that noncircularity plays only a secondary role in achieving high twistiness-to-bendiness ratios. The main player has to be tuning of the material properties, the relevant elastic moduli, rather than geometric factors, the second moments of area.

\section{Making the Most of Material Anisotropy}

Organisms use composite materials to resist all but the simplest tensile loads. Most if not all of these composites are anisotropic. One can crack a brick in any direction, but a log splits only with the grain. The elevated values of EI/GJ just noted clearly depend on material anisotropy, although in no case have the specific details yet been worked out. Thus, entering data for the elastic moduli into the textbook formula relating the two to the Poisson ratio for isotropic materials

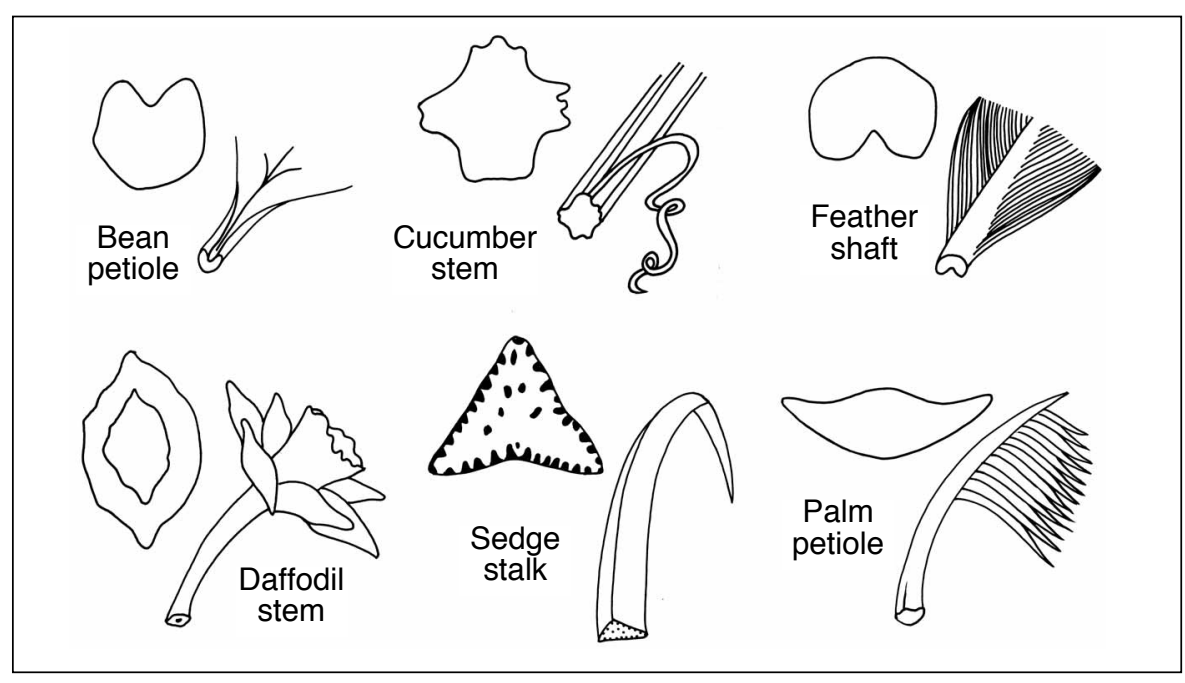

Figure 5. Cantilever structures with noncircular cross sections that experience torsion as a result of crosswise flow. 
gives wildly unreasonable values for that latter ratio- 6 or 7 for tree trunks and even higher for dried and sliced wood.

The same point emerges from a look at design simulations. One can make a computer describe a structure in which, for a designated load, stress is everywhere constant and the material of the structure is thereby optimally employed. For some biological structures, reality reasonably resembles the results. For others, though, the simulations diverge from what we see. Thus a tiger claw, based on the assumption of constant stress, turns out to be unreasonably blunt. ${ }^{12}$ Stresses on a real claw must increase from base to tip, and, given their particular shapes, the same most likely holds for many other claws, fangs, and even (in invertebrates) jaws. Do animals simply tolerate greater tipward fragility? In several cases we know that that is not the case. The jaws of nereid and glycerid polychaete worms (which look to us more like claws than jaws) are stiffened with metallic salts, zinc in the former and copper in the latter. In both groups, the proportion of those stiffening and presumably strengthening compounds increases out toward their tips. $^{13,14}$ So a material-minimizing design will not be one that assumes constant stress-in a simulation, stress must be made (or at least allowed) to increase tipward. And that will permit a more realistically sharp jaw.

Not only do the metallic salts occur in higher concentration near the sharp ends of the fang-like jaws, but, according to recent work on a glycerid, coppercontaining fibers are more concentrated in the middle of the jaws than nearer the surface. Thus, stiffness increases from outside inward, and the (relative) softness of the surface reduces the chance of crack initiation. A worm's jaws must often close on a bit of hard gravel rather than softer edible material, so reducing the chance of contact damage might matter a great deal. Inadvertently bite on a pebble instead of a peanut, and you are likely to break a tooth. ${ }^{15}$ Of course, worms do not grind with their jaws as do you with your surface-hardened teeth.

\section{The Promise of Biomimetics}

The general point is that nature neither holds nor should be expected to hold any natural superiority, and it provides neither comfort nor example for engineerbashers. Our technology does all kinds of things with few or no natural analogues, even things we think might be useful to other organisms. Even ancient humans repeatedly invented things without the benefit of natural models-metal imple- ments, baked ceramics for pots and bricks, fabrics for clothing, and so forth. We should not view our suspension bridges as intrinsically less effective than nature's vines, our electric wires less than nerve axons, turbines less than muscles, or ship propellers less than fish tails.

... nature's technology
differs from our own along a
host of axes, and in those
differences lies the promise
of biomimetics.

But neither should we ignore what organisms have evolved. We have much to learn simply because nature, for whatever reasons, has done things differently. Even if the superiority of nature is a myth, such a vastly different technology can only be a gold mine of stimulation, even inspiration. So what if nature's splendid achievements in multicomponent composite materials reflect an ignorance of metallurgy? Bone, tooth, wood, and the chitin of insect skeletons still help us understand how mechanical behavior derives from chemical composition and supermolecular structure.

Close imitation may yield less; certainly it has had no glorious history, despite a pervasive mythology to the contrary. Should we try to synthesize spider silkassuming we have in mind some application for this peculiar stuff? We are not good at making sequence-specific heteropolymers of amino acids by the gram, much less the ton-nature's manufacturing methods and ours bear even less resemblance to each other than do their products. But we have no reason to believe that silk's properties can only be realized by that route; indeed, we have every reason to believe that nature uses that approach simply because, at least in the animal kingdom, that is the default way to make a tensile material. So we might contrive a pseudosilk of a kind that can be more efficiently made by our synthetic technology. Making true spider silk might make sense if we see a market sufficiently price-insensitive or undemanding of large quantities to justify co-opting the proteinproducing facility of some organism. But, again, we can learn from nature-spider silk shows us what is possible and might tell us something we do not already know about how polymeric structure translates into functional properties.

Profit and knowledge might just emerge from asking how nature manages its mechanical affairs so well when lacking so many of our favorite devices. Better yet, nature's evolved technology represents the only one other than our own to which we have access. Where nature does what we do, one suspects intrinsic technical superiority for a scheme. Where nature adds tricks that we have yet to try, we get ideas and a rare chance for cheap analysis that precedes expensive synthesis. Where nature does something different-as in its uses of flexibility - one gets the hint of multiple worlds, even ones as yet explored by neither technology.

\section{Acknowledgments}

The author thanks the Materials Research Society for the invitation to give the talk, at the December 2002 meeting in Boston, that led to this article. (In much less compact form, its content forms part of Reference 2.) Annette deFerrari drew the freehand illustrations.

\section{References}

1. S. Vogel, Cats' Paws and Catapults: Mechanical Worlds of Nature and People (W.W. Norton, New York, 1998).

2. S. Vogel, Comparative Biomechanics: Life's Physical World (Princeton University Press, Princeton, NJ, 2003)

3. J.E. Gordon, Structures: Or, Why Things Don't Fall Down (Plenum Press, New York, 1978).

4. M.A.R. Koehl, J. Exp. Biol. 69 (1977) p. 127.

5. C.D. Harvell and M. LaBarbera, Biol. Bull. 168 (1985) p. 312.

6. A.S. Johnson, Biol. Bull. 185 (1993) p. 10.

7. E.C. Bell and J.M. Gosline, J. Exp. Biol. 199 (1996) p. 1005.

8. S. Vogel, Life in Moving Fluids: The Physical Biology of Flow, 2d ed. (Princeton University Press, Princeton, NJ, 1994).

9. S. Vogel, J. Exp. Bot. 43 (1992) p. 1527.

10. A.R. Ennos, Ann. Bot. 72 (1993) p. 123.

11. S.A. Etnier and S. Vogel, Amer. J. Bot. 87 (2000) p. 29.

12. C. Mattheck, Design in Nature: Learning from Trees (Springer-Verlag, Berlin, 1998).

13. G.W. Bryan and P.E. Gibbs, J. Mar. Biol. Assoc. U.K. 60 (1980) p. 641.

14. P.E. Gibbs and G.W. Bryan, J. Mar. Biol. Assoc. U.K. 60 (1980) p. 205

15. H.C. Lichtenegger, T. Schöbert, M.H. Bartl, H. Waite, and G.D. Stucky, Science 298 (2002) p. 389.

Steven Vogel is James B. Duke Professor of Biology at Duke University. He is the author of several books, aimed at the general reader, in which he compares the mechanical technologies of nature with those fabricated by scientists; these books include Cats' Paws and Catapults: Mechanical Worlds of Nature and People (W.W. Norton, New 


\section{MATERIAL MATTERS}

York, 1998), Life's Devices (Princeton University Press, Princeton, NJ, 1988), and Comparative Biomechanics: Life's Physical World (Princeton University Press, Princeton, NJ, 2003). Among Vogel's professional recognitions are the Irving and Jean Stone Prize for Science Writing for Public
Understanding (1990) and the Trinity College (Duke University) Distinguished Teaching Award (1986). He was named a fellow of the American Association for the Advancement of Science in 1983. He was recently honored as Kieval Lecturer by Cornell University (1999), Richard C.
Schultz Lecturer by the Rochester Museum and Science Center (2001), and Donald Haragan Lecturer by Texas Tech University (2001). Vogel's professional memberships include the American Society of Biomechanics, and he has numerous publications in peer-reviewed journals. Vogel can be reached by e-mail at svogel@duke.edu. 